Research Article

\title{
Screening and Characterizing Tyrosinase Inhibitors from Salvia miltiorrhiza and Carthamus tinctorius by Spectrum-Effect Relationship Analysis and Molecular Docking
}

\author{
Ya-Li Wang, ${ }^{1,2}$ Guang Hu $\mathbb{D}^{1},{ }^{1}$ Qian Zhang, ${ }^{2}$ Yu-Xiu Yang, ${ }^{2}$ Qiao-Qiao Li, ${ }^{2}$ \\ Yuan-Jia $\mathrm{Hu}\left(\mathbb{1},{ }^{3}\right.$ Hua Chen, ${ }^{2}$ and Feng-Qing Yang $\mathbb{C}^{2}$ \\ ${ }^{1}$ School of Pharmacy and Bioengineering, Chongqing University of Technology, Chongqing 400054, China \\ ${ }^{2}$ School of Chemistry and Chemical Engineering, Chongqing University, Chongqing 401331, China \\ ${ }^{3}$ State Key Laboratory of Quality Research in Chinese Medicine, Institute of Chinese Medical Sciences, \\ University of Macau, Macau
}

Correspondence should be addressed to Guang Hu; foxhu8201@hotmail.com and Feng-Qing Yang; fengqingyang@cqu.edu.cn Received 13 February 2018; Accepted 1 April 2018; Published 9 May 2018

Academic Editor: Jaroon Jakmunee

Copyright (C) 2018 Ya-Li Wang et al. This is an open access article distributed under the Creative Commons Attribution License, which permits unrestricted use, distribution, and reproduction in any medium, provided the original work is properly cited.

Tyrosinase (TYR) is a rate-limiting enzyme in the synthesis of melanin, while direct TYR inhibitors are a class of important clinical antimelanoma drugs. This study established a spectrum-effect relationship analysis method and high-performance liquid chromatography-mass spectrometry (LC-MS) analysis method to screen and identify the active ingredients that inhibited TYR in Salvia miltiorrhiza-Carthamus tinctorius (Danshen-Honghua, DH) herbal pair. Seventeen potential active compounds (peaks) in the extract of DH herbal pair were predicted, and thirteen of them were tentatively identified by LC-MS analysis. Furthermore, TYR inhibitory activities of five pure compounds obtained from the DH herbal pair were validated in the test in which kojic acid served as a positive control drug. Among them, three compounds including protocatechuic aldehyde, hydroxysafflor yellow A, and tanshinone IIA were verified to have high TYR inhibitory activity (IC50 value of 455, 498, and $1214 \mu \mathrm{M}$, resp.) and bind to the same amino acid residues in TYR catalytic pocket according to the results of the molecular docking test. However, the other two compounds lithospermic acid and salvianolic acid A had a weak effect on TYR, as they do not combine with the active amino acid residues or act on the active center of TYR. Therefore, the developed methods (spectrum-effect relationship analysis and molecular docking) could be used to effectively screen TYR inhibitors in complex mixtures such as natural products.

\section{Introduction}

Tyrosinase (TYR) belongs to the type 3 copper protein family containing dinuclear copper ions and widely exists in nature from microorganisms to humans [1]. It is a critical enzyme in the synthesis process of melanin pigments, catalyzing orthohydroxylation of monophenols to $o$-diphenols and then to the corresponding o-quinones [2]. However, overproduction of melanin pigments becomes a problem in the cosmetic and clinical points of view, such as melasma, freckles, and melanosis $[3,4]$. Due to the importance of TYR during the synthesis of melanin, blocking the activity of TYR is one of the ideal strategies to treat melanin pigment diseases currently. To date, arbutin and kojic acid are the most commonly used tyrosinase inhibitors, which often serve as positive control drugs $[5,6]$. However, traditional synthetic or microbial origin tyrosine inhibitors have some drawbacks, such as long-term contact of hydroquinone can lead to skin cancer, dermatitis, and other diseases $[7,8]$. In reality, it is reported that the polyphenols and flavonoids isolated from natural plants have significant tyrosinase inhibition effect and less potential side effects $[9,10]$. From this point of view, it is of great importance to screen TYR inhibitors from natural products.

Salviae Miltiorrhizae Radix et Rhizoma (Danshen in Chinese, DS) is the dried root or rhizome of Salvia miltiorrhiza 
Bunge, in which the main components are phenolic acids and diterpenes [11]. Carthami flos (Honghua in Chinese, $\mathrm{HH}$ ), the dried flower of Carthamus tinctorius L., is generally composed of flavonoids, fatty acids, volatile oils, and polysaccharides [12]. In previous reports, the inhibitory effect of Salvia miltiorrhiza and Carthamus tinctorius on tyrosinase has been validated [13-15]; however, the active constituents with tyrosinase inhibition activity have not been clearly reported yet. Therefore, in this study, a spectrum-effect analysis method is developed to screen the active constituents that inhibit tyrosinase in Danshen-Honghua (DH) herbal pair. The spectrum-effect relationship analysis combines the chemical compositions of the fingerprint of natural products with the results of the efficacy, and is originally used to develop control standards that can truly reflect the inherent quality of products [16]. Furthermore, spectrum-effect analysis is also used to screen the active components from natural products [17]. In reality, spectrum-effect analysis shows some positive features such as reliability, time-saving capacity, and simple operation $[18,19]$.

In this study, the inhibition effect of $\mathrm{DH}$ herbal pair and single drug on tyrosinase was compared first. Then, the components in the $\mathrm{DH}$ herbal pair are analyzed and identified by HPLC analysis. Third, the active components in $\mathrm{DH}$ herbal pair were predicted by spectrum-effect analysis, and their structures were identified by LC-MS analysis. Furthermore, the TYR inhibition activities of the predicted compounds were evaluated in an in vitro model. Finally, molecular docking, which is a method of drug design through the characterization of the receptor and the interaction between the receptor and the drug molecules, and binding mode and affinity prediction [20], was used to confirm the binding sites of compounds with tyrosinase and to predict several possible TYR inhibitors which possess similar structure to the screened active compounds by molecular docking.

\section{Materials and Methods}

2.1. Chemicals and Materials. Tyrosinase (MW $128 \mathrm{kDa})$ from Agaricus bisporus, kojic acid ( $\geq 98 \%$ ), and L-tyrosine were purchased from Sigma-Aldrich (St. Louis, MO, USA). Tyrosinase, kojic acid, and L-tyrosine were dissolved in $50 \mathrm{mM}$ sodium phosphate buffer ( $\mathrm{pH}$ 6.8) before use. The reference compounds protocatechuic aldehyde, hydroxysafflor yellow A, tanshinone IIA, lithospermic acid, and salvianolic acid A ( $\geq 98 \%$, determined by HPLC) were obtained from PUSH Bio-Technology Co., Ltd. (Chengdu, China). HPLC-grade acetonitrile and formic acid were obtained from Beijing InnoChem Science \& Technology Co., Ltd. (Beijing, China). All of the experimental water was purified by water purification system (ATSelem 1820A, Antesheng Environmental Protection Equipment Co., Ltd., Chongqing, China). All other chemicals and solvents, unless otherwise specified, were guaranteed reagent grade and purchased from SigmaAldrich Chemical Co. LLC. (St. Louis, MO, USA).

Crude drugs of Danshen and Honghua were both purchased from Chongqing Heping Pharmacy Co., Ltd. (Chongqing, China), in June 2017. The voucher specimens of
Salvia miltiorrhiza Bunge (number SM2017090101) and Carthamus tinctorius L. (number CF2017090101) were deposited at the Pharmaceutical Engineering Laboratory in School of Chemistry and Chemical Engineering, Chongqing University, Chongqing, China.

2.2. Preparation of DH Extracts and Stock Solutions. All the dried raw DS and $\mathrm{HH}$ were pulverized and griddled through 50 mesh sieves (about $0.29 \mathrm{~mm}$ ) prior to extraction. Seven different proportions of the herbs were prepared with ratios of $1: 1,2: 1,3: 1,5: 1,1: 5,1: 3$, and $1: 2(\mathrm{~g} / \mathrm{g})$ DS to $\mathrm{HH}$, respectively. $20 \mathrm{~g}$ of $\mathrm{DS}$ and $\mathrm{HH}$ mixed powder was extracted with $200 \mathrm{~mL}$ water in a glass-stoppered conical flask at $75^{\circ} \mathrm{C}$ for $1.5 \mathrm{~h}$. After extraction, the mixture was filtered through gauze, and the residue was collected and extracted with the above process for a second time. The two filtrates were combined and evaporated in a rotary evaporator (ZFQ 85A, Shanghai Medical Instrument Special Factory, Shanghai, China) at $55^{\circ} \mathrm{C}$ under reducing pressure to remove the solvent. The extracts were further dried by lyophilization with freezing-drying system (DZF-6050, Shanghai Jing Hong Laboratory Instrument Co., Ltd., Shanghai, China) to obtain the $\mathrm{DH}$ extracts at a yield of about $25 \%$ (w/w, dried extract/crude herb). All pre- and postdilution solutions were stored at $4^{\circ} \mathrm{C}$. Before HPLC analysis, the sample solutions were filtered through a $0.22 \mu \mathrm{m}$ nylon membrane filter (Shanghai Titan Scientific Co., Ltd., Shanghai, China).

The reference substances protocatechuic aldehyde, hydroxysafflor yellow A, tanshinone IIA, lithospermic acid, and salvianolic acid A and a positive control (kojic acid) were all prepared by dissolving the respective substance in methanol solution and diluted with PBS (50 mM, pH 6.8) to the required concentrations for TYR inhibitory and binding assay, respectively. All the solutions were stored at $4^{\circ} \mathrm{C}$ in dark before use.

2.3. HPLC and LC-MS Analysis. HPLC analysis was performed on an Agilent 1260 series liquid chromatograph system (Agilent Technologies, Palo Alto, CA, USA), which was equipped with a vacuum degasser, a binary pump, an autosampler, and a diode array detector (DAD), controlled by Agilent ChemStation software. An Agilent Zorbax SB-Aq column $(250 \mathrm{~mm} \times 4.6 \mathrm{~mm}, 5 \mu \mathrm{m})$ preceded by a Zorbax SB- $\mathrm{C}_{18}$ guard column $(12.5 \times 4.6 \mathrm{~mm}, 5 \mu \mathrm{m})$ was adopted for the analysis. The mobile phase consisted of solvent A $(0.1 \%$ formic acid aqueous solution) and solvent B (acetonitrile) using a gradient elution, which was programmed as follows: $5 \% \mathrm{~B}$ at $0-2 \mathrm{~min}, 5 \%-15 \% \mathrm{~B}$ at $2-10 \mathrm{~min}, 15 \%-22 \% \mathrm{~B}$ at $10-24 \mathrm{~min}, 22 \%-29 \% \mathrm{~B}$ at $24-35 \mathrm{~min}, 29 \%-37 \% \mathrm{~B}$ at $35-$ $42 \mathrm{~min}$, and $37 \%-5 \% \mathrm{~B}$ at $42-47 \mathrm{~min}$. The mobile phase was set at a flow rate of $1.0 \mathrm{~mL} / \mathrm{min}$ with $10 \mu \mathrm{L}$ per sample injection. The UV detection wavelength was set at $280 \mathrm{~nm}$, and the column temperature was conditioned at $37^{\circ} \mathrm{C}$.

Shimadzu LC/MS-MS 8060 electrospray ionization-mass spectrometer (ESI-MS), consisting of a Triple Quadruple Detector (TSQ) as the mass detector (Shimadzu, Kyoto, Japan) and coupled with HPLC, was used for LC-MS identification. The LC conditions were the same as 
described previously. The ESI-MS conditions were as follows: the ESI was used in both positive and negative mode; nitrogen gas was used for desolvation at a flow rate of $3 \mathrm{~L} / \mathrm{min}$ at $250^{\circ} \mathrm{C}$; the temperature and flow rate of drying gas were set under $400^{\circ} \mathrm{C}$ and $10 \mathrm{~L} / \mathrm{min}$, respectively; the cone voltage was (+) 20 and (-) $20 \mathrm{~V}$; MS data were recorded in the full-scan mode $(\mathrm{m} / \mathrm{z} 50-1500)$, and $\mathrm{MS}^{2}$ data were recorded in the range of $m / z 50-1200$.

2.4. TYR Inhibitory Activity Assay. The enzyme assay was performed in 96-well Corining Costar plates (Corning Incorporated, USA). $50 \mu \mathrm{L}$ test solution and $50 \mu \mathrm{L}$ TYR solution $(800 \mathrm{U} / \mathrm{mL})$ were mixed and incubated for $10 \mathrm{~min}$ at room temperature. After the incubation, $100 \mu \mathrm{L}(1 \mathrm{mg} / \mathrm{mL})$ of L-tyrosine in PBS ( $\mathrm{pH}$ 6.8) buffer as chromogenic substrate was added to start the reaction. The absorbance was monitored at $490 \mathrm{~nm}$ every $30 \mathrm{~s}$ for 10 min with an iMark ${ }^{\mathrm{TM}}$ Microplate Absorbance Reader (Bio-Rad Laboratories, Inc., USA). PBS ( $50 \mathrm{mM}, \mathrm{pH} 6.8$ ) buffer was prepared as blank control, and kojic acid was used as positive control. TYR inhibition activity was expressed as the inhibitory percentage of TYR:

$$
\begin{aligned}
\text { Inhibition percentage }(\%)= & \frac{(d A / d t)_{\text {blank }}-(d A / d t)_{\text {sample }}}{(d A / d t)_{\text {blank }}} \\
& \times 100 \%,
\end{aligned}
$$

where $(d A / d t)_{\text {blank }}$ and $(d A / d t)_{\text {sample }}$ are the reaction rate of the blank and sample group, respectively. All trials were independently performed in triplicates, and the results were shown with mean value of the triplicate observations.

2.5. Spectrum-Effect Relationship Analysis. Spectrum-effect analysis was performed by transferring DH fingerprint peak area and TYR inhibition activity test results into SPSS software for canonical correlation analysis (CCA). The optimized HPLC fingerprints of seven ratios DH samples were calculated and generated by professional software named "Similarity Evaluation System for Chromatographic Fingerprint of Traditional Chinese Medicine" composed by Chinese Pharmacopoeia Committee (Version 2012). CCA was used to assess the spectrum-effect relationships between the areas of 86 peaks in fingerprint and the TYR inhibition ratios.

2.6. In Silico Molecular Docking of TYR and Identified Active Compounds. Auto Dock 4.2 program (The Scripps Research Institute, La Jolla, CA, USA) was employed for in silico molecular docking study to validate the binding potency of the compounds to TYR [21]. The docking operation was performed according to the following steps. First, the crystal structure file of TYR (Agaricus bisporus mushroom tyrosinase) complex (PDB ID $=2 \mathrm{y} 9 \mathrm{x})$ was downloaded [22] The dimension grid box $(90 \AA \times 90 \AA \times 102 \AA)$ and the grid spacing of $0.619 \AA$ were defined to enclose the active site. Second, the ligand was deleted using UCSF Chimera, and
TABLE 1: Inhibition effect of DH extracts on tyrosinase with different ratios $(n=9)$.

\begin{tabular}{lc}
\hline Sample & Inhibition rate (\%) \\
\hline Blank $^{\mathrm{a}}$ & $0.06 \pm 0.01$ \\
Kojic acid $^{\mathrm{b}}$ & $76.83 \pm 0.24$ \\
DH $1: 0^{\mathrm{b}}$ & $26.55 \pm 0.17$ \\
DH $0: 1^{\mathrm{b}}$ & $34.70 \pm 0.98$ \\
DH $1: 1^{\mathrm{b}}$ & $42.19 \pm 0.10$ \\
DH $2: 1^{\mathrm{b}}$ & $29.93 \pm 0.32$ \\
DH $3: 1^{\mathrm{b}}$ & $40.53 \pm 0.28$ \\
DH $5: 1^{\mathrm{b}}$ & $46.84 \pm 0.30$ \\
DH $1: 5^{\mathrm{b}}$ & $16.15 \pm 1.05$ \\
DH $1: 3^{\mathrm{b}}$ & $25.72 \pm 0.17$ \\
DH $1: 2^{\mathrm{b}}$ & $38.90 \pm 0.09$ \\
\hline
\end{tabular}

${ }^{\mathrm{a}}$ Concentration: PBS (50 mM, pH6.8); ${ }^{\mathrm{b}}$ concentration: $500 \mu \mathrm{g} / \mathrm{mL}$.

unnecessary water molecules were removed, and hydrogen atoms were added [23]. Third, the 3D chemical structure of investigated compounds was drawn by using Microsoft office 3D and output in PDB format with minimized energy.

With the aim of docking with Autodock Vina, the grid size was set to $(x, y, z)=(50,50,50)$ and the grid center was set to $(x, y, z)=(10.044,28.706,43.443)$. In each simulation process, progress with default parameters run from Autogrid and Autodock. Lamarckian genetic algorithm (LGA) was used to find the most favorable ligand binding orientations, and the number of LGA runs is equal to 50 . The interaction figures were generated, and the results of docking were recorded with binding energies and bonded residues.

2.7. Statistical Analysis. All data are presented as mean \pm standard deviations (SD) of at least three different experiments. The statistical analysis was performed with SPSS (version 24, SPSS, Inc., Chicago, IL, USA).

\section{Results and Discussion}

3.1. Effects of DH Extracts on Tyrosinase Activity. As shown in Table 1 and Figure 1, PBS served as a blank control, while kojic acid served as a positive control. Some DH herbal pair extracts $(1: 5$ and $1: 3)$ showed a weaker inhibitory effect than single herbal extracts when the concentrations of tyrosinase and the sample were kept constant. However, other DH herbal pair extracts $(1: 1,3: 1,5: 1$, and $1: 2)$ displayed a stronger inhibitory effect than single herbal extracts, which indicated that a synergistic effect of the herbal pair may occur on the inhibition of tyrosinase activity. Therefore, $\mathrm{DH}$ herbal pair was used as the research object for screening their tyrosinase inhibitors.

In order to obtain the best screening performance for active compounds in the complex matrix, some important parameters of the method including incubation time, TYR concentration, and sample concentration should be optimized. According to previous research, the incubation time might be controlled at the range of $30-120 \mathrm{~min}$ because a short incubation time (less than $30 \mathrm{~min}$ ) might prevent the identification of target molecules which are not firmly bound to TYR, while a long incubation time (about 120 


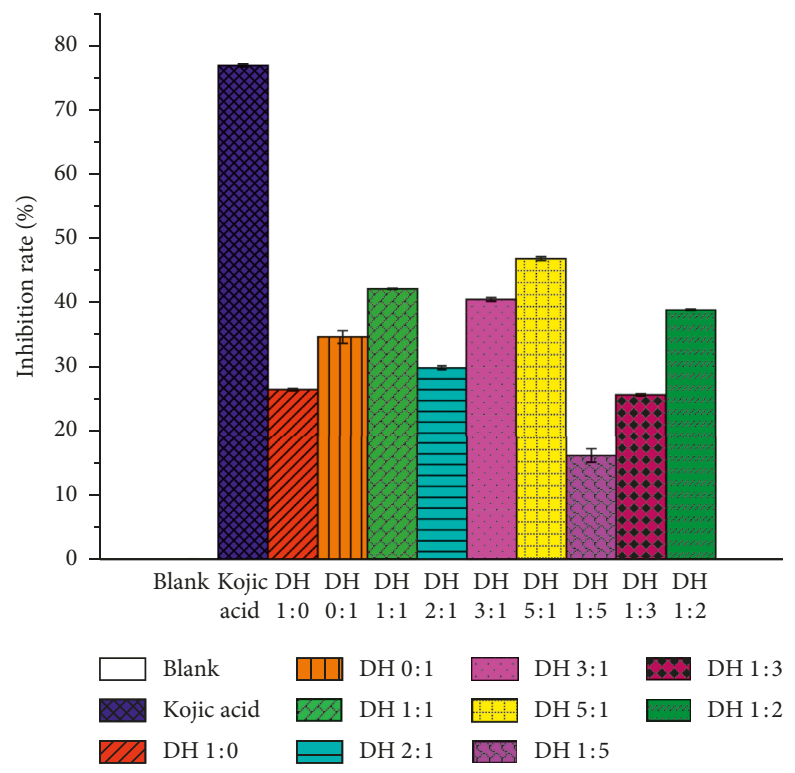

Figure 1: Different ratios of DH herbal pair on tyrosinase inhibition effects.

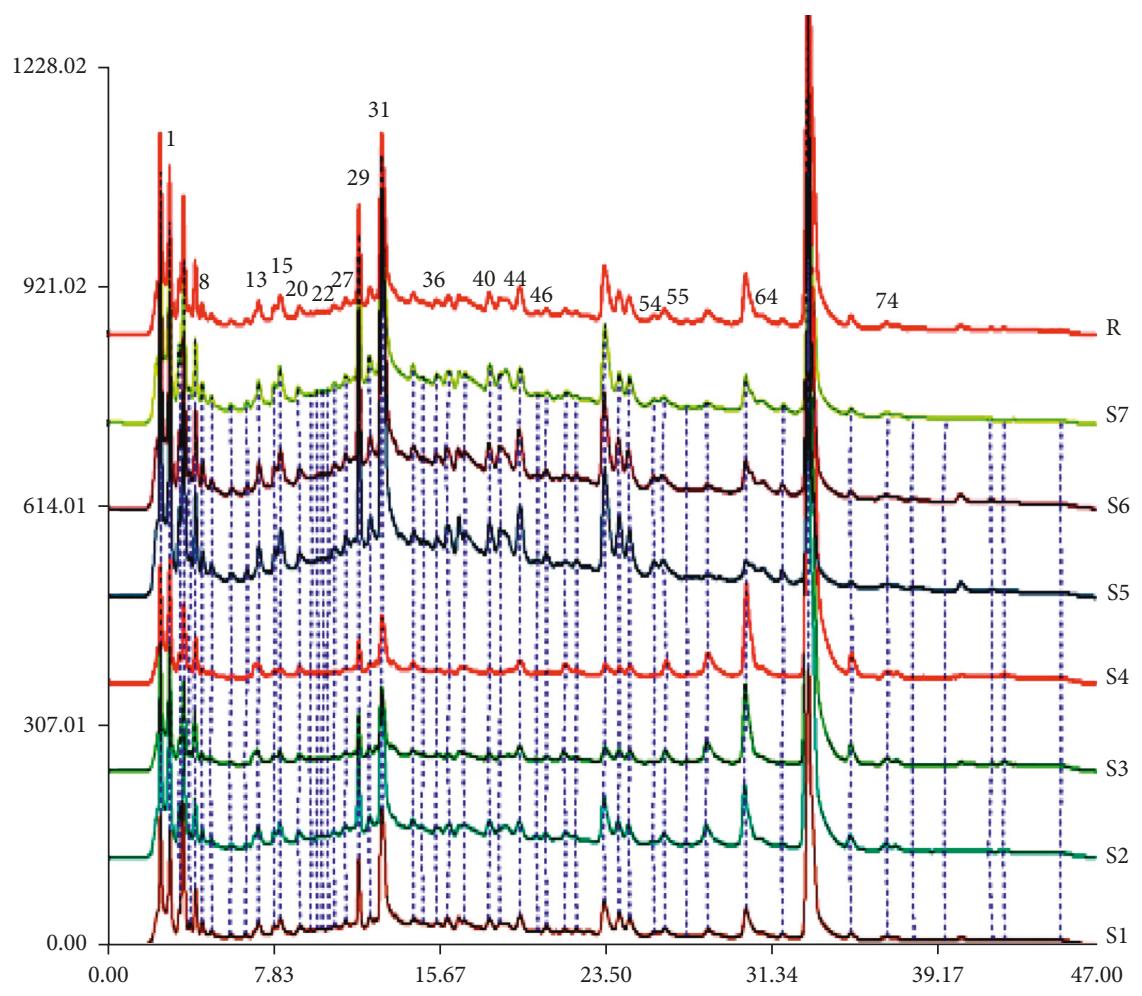

FIGURE 2: HPLC calibration fingerprints of DH herbal pair with different ratios. The chromatograms of S1-S7 are represented as follows: DH 1:1 (S1); DH 2:1 (S2); DH 3:1 (S3); DH 5:1 (S4); DH 1:5 (S5); DH 1:3 (S6); DH 1:2 (S7); control map (R).

minutes) would not make significant influence on the screening result $[24,25]$. After investigation, a proper TYR concentration $(800 \mathrm{U} / \mathrm{mL})$ increased the sensitivity and number of bioactive constituents detected in the sample; meanwhile, the inhibition effect of tyrosinase is most stable by the incubation time of $40 \mathrm{~min}$ (data not shown). Therefore, a sufficient incubation time (40 min) and a sufficient TYR concentration $(800 \mathrm{U} / \mathrm{mL})$ were used in this study.

3.2. Spectrum-Effect Relationship Analysis. The optimized HPLC fingerprints of DH samples with seven ratios are shown in Figure 2. A total of 86 peaks involved were detected in the 
TABLE 2: Correlation coefficients between chromatogram peaks and inhibition rates.

\begin{tabular}{lcccccccccccccc}
\hline Peak number & 1 & 2 & 3 & 4 & 5 & 6 & 7 & 8 & 9 & 10 & 11 & 12 & 13 \\
Inhibition rate & $0.940^{*}$ & -0.702 & -0.695 & -0.501 & -0.753 & -0.769 & -0.677 & $0.812^{*}$ & -0.721 & -0.775 & -0.790 & -0.351 & $0.807^{*}$ \\
Peak number & $\mathbf{1 4}$ & $\mathbf{1 5}$ & $\mathbf{1 6}$ & $\mathbf{1 7}$ & $\mathbf{1 8}$ & $\mathbf{1 9}$ & $\mathbf{2 0}$ & $\mathbf{2 1}$ & $\mathbf{2 2}$ & $\mathbf{2 3}$ & $\mathbf{2 4}$ & $\mathbf{2 5}$ & $\mathbf{2 6}$ \\
Inhibition rate & 0.510 & $-0.878^{*}$ & -0.742 & -0.632 & -0.713 & 0.714 & $0.919^{*}$ & -0.746 & $0.903^{*}$ & -0.129 & -0.615 & -0.233 & -0.623 \\
Peak number & 27 & 28 & 29 & 30 & 31 & 32 & 33 & 34 & 35 & 36 & 37 & 38 & 39 \\
Inhibition rate & $0.952^{*}$ & -0.544 & $0.908^{*}$ & 0.650 & $0.900^{*}$ & -0.397 & -0.015 & -0.466 & 0.321 & $-0.824^{*}$ & -0.795 & -0.726 & -0.762 \\
Peak number & $\mathbf{4 0}$ & $\mathbf{4 1}$ & $\mathbf{4 2}$ & $\mathbf{4 3}$ & $\mathbf{4 4}$ & $\mathbf{4 5}$ & $\mathbf{4 6}$ & $\mathbf{4 7}$ & $\mathbf{4 8}$ & $\mathbf{4 9}$ & $\mathbf{5 0}$ & $\mathbf{5 1}$ & $\mathbf{5 2}$ \\
Inhibition rate & $0.892^{*}$ & -0.571 & -0.790 & -0.785 & $-0.867^{*}$ & -0.745 & $0.891^{*}$ & -0.640 & -0.746 & -0.708 & 0.135 & -0.753 & -0.451 \\
Peak number & $\mathbf{5 3}$ & $\mathbf{5 4}$ & $\mathbf{5 5}$ & $\mathbf{5 6}$ & $\mathbf{5 7}$ & $\mathbf{5 8}$ & $\mathbf{5 9}$ & $\mathbf{6 0}$ & $\mathbf{6 1}$ & $\mathbf{6 2}$ & $\mathbf{6 3}$ & $\mathbf{6 4}$ & $\mathbf{6 5}$ \\
Inhibition rate & -0.627 & $-0.943^{*}$ & $-0.929^{*}$ & -0.759 & -0.778 & -0.037 & -0.769 & 0.591 & -0.621 & -0.741 & 0.786 & $0.824^{*}$ & -0.725 \\
Peak number & $\mathbf{6 6}$ & $\mathbf{6 7}$ & $\mathbf{6 8}$ & $\mathbf{6 9}$ & $\mathbf{7 0}$ & $\mathbf{7 1}$ & $\mathbf{7 2}$ & $\mathbf{7 3}$ & $\mathbf{7 4}$ & $\mathbf{7 5}$ & $\mathbf{7 6}$ & $\mathbf{7 7}$ & $\mathbf{7 8}$ \\
Inhibition rate & -0.667 & -0.772 & 0.596 & 0.618 & 0.124 & 0.704 & -0.712 & 0.654 & $0.887^{*}$ & 0.215 & 0.361 & 0.371 & 0.718 \\
Peak number & $\mathbf{7 9}$ & $\mathbf{8 0}$ & $\mathbf{8 1}$ & $\mathbf{8 2}$ & $\mathbf{8 3}$ & $\mathbf{8 4}$ & $\mathbf{8 5}$ & $\mathbf{8 6}$ & & -0.123 & -0.512 & & & \\
Inhibition rate & 0.610 & 0.646 & -0.733 & 0.565 & -0.717 & 0.279 & -0.123 & & & \\
\hline
\end{tabular}

Note. Pearson correlation, "r" represents the relevant strength; ${ }^{*} 0.8 \leq|r| \leq 1$ means Very significant correlation.

TABLE 3: HPLC-MS/MS data of 17 predicted active compounds from DH herbal pair.

\begin{tabular}{|c|c|c|c|c|c|c|}
\hline Peak No. & $t_{\mathrm{R}}(\min )$ & MW & $\operatorname{MS}^{1}(m / z)$ & $\mathrm{MS}^{2}(m / z)$ & Formula & Structural identification \\
\hline 1 & 3.087 & 138 & 138.05 & $92 ; 78 ; 65$ & $\mathrm{C}_{7} \mathrm{H}_{6} \mathrm{O}_{3}$ & Protocatechuic aldehyde \\
\hline 8 & 3.112 & 198 & 197.05 & 178 & $\mathrm{C}_{9} \mathrm{H}_{10} \mathrm{O}_{5}$ & Danshensu \\
\hline 13 & 6.491 & 165 & 166.09 & 120 & $\mathrm{C}_{9} \mathrm{H}_{11} \mathrm{NO}_{2}$ & Phenylalanine \\
\hline 15 & 7.108 & - & - & - & - & Unknown \\
\hline 20 & 7.952 & 294 & 295.15 & $277 ; 249$ & $\mathrm{C}_{19} \mathrm{H}_{18} \mathrm{O}_{3}$ & Tanshinone IIA \\
\hline 22 & 8.951 & 180 & 179.10 & 135 & $\mathrm{C}_{9} \mathrm{H}_{8} \mathrm{O}_{4}$ & Caffeic acid \\
\hline 27 & 11.207 & - & - & - & - & Unknown \\
\hline 29 & 12.386 & 788 & 787.40 & $625 ; 505 ; 463 ; 301$ & $\mathrm{C}_{33} \mathrm{H}_{40} \mathrm{O}_{22}$ & 6-Hydroxykaempferol-3,6,7-O- $\beta$-D-glucoside \\
\hline 31 & 12.771 & 612 & 611.30 & $491 ; 473 ; 403 ; 353 ; 325 ; 283 ; 205$ & $\mathrm{C}_{27} \mathrm{H}_{32} \mathrm{O}_{16}$ & Hydroxysafflor yellow A \\
\hline 36 & 14.094 & 640 & 639.30 & $463 ; 362 ; 300 ; 255 ; 139$ & - & Unknown \\
\hline 40 & 17.091 & 772 & 773.35 & $695 ; 672 ; 303 ; 187 ; 112$ & $\mathrm{C}_{33} \mathrm{H}_{40} \mathrm{O}_{21}$ & $\begin{array}{l}\text { 6-Hydroxykaempferol 3-O-rutinoside-6-O- } \\
\text { glucoside }\end{array}$ \\
\hline 44 & 21.352 & - & - & - & - & Unknown \\
\hline 46 & 23.969 & 1044 & 1043.45 & $1025 ; 923 ; 863 ; 764 ; 593 ; 449$ & $\mathrm{C}_{48} \mathrm{H}_{52} \mathrm{O}_{26}$ & Anhydrosafflor yellow B \\
\hline 54 & 29.882 & 360 & 359.25 & $179 ; 161 ; 133$ & $\mathrm{C}_{18} \mathrm{H}_{16} \mathrm{O}_{8}$ & Rosmarinic acid \\
\hline 55 & 31.813 & 538 & 537.25 & $295 ; 253 ; 203$ & $\mathrm{C}_{27} \mathrm{H}_{22} \mathrm{O}_{12}$ & Lithospermic acid \\
\hline 64 & 32.528 & 494 & 493.25 & 295 & $\mathrm{C}_{26} \mathrm{H}_{22} \mathrm{O}_{10}$ & Salvianolic acid A \\
\hline 74 & 36.747 & 718 & 717.35 & $673 ; 617 ; 519 ; 321$ & - & Salvianolic acid E \\
\hline
\end{tabular}

calculation of spectrum-effect relationship. CCA (canonical correlation analysis) was used to assess the spectrum-effect relationship between the areas of 86 peaks and the main parameters (inhibition rate), and the results are shown in Table 2. As suggested by the correlation coefficients, the highly relevant peaks were $1,8,13,15,20,22,27,29,31,36,40,44,46,54,55$, 64 , and 74 with Pearson relational grade more than 0.8 . In other words, these 17 peaks might be the main active components of herbal pair for inhibiting tyrosinase, and further studies were performed to identify the structures of these peaks to confirm their bioactivities.

\subsection{Identification of the Potential TYR-Targeted Compound} by LC-MS Analysis. HPLC-MS/MS analysis was used to identify the chemical structures of compounds in $\mathrm{DH}$ extracts. Based on the fragmentation behaviors, retention time and MS data (Table 3) of the peaks in the test samples, 13 compounds (protocatechuic aldehyde, danshensu, phenylalanine, tanshinone IIA, caffeic acid, 6-hydroxykaempferol-3,6,7$O-\beta$-D-glucoside, hydroxysafflor yellow A, rosmarinic acid, anhydrosafflor yellow B, 6-hydroxykaempferol 3-O-rutinoside6-O-glucoside, lithospermic acid, salvianolic acid A, and salvianolic acid E) were tentatively identified, and the structures of these compounds are shown in Figure 3. Five pure reference compounds including protocatechuic aldehyde, tanshinone IIA, hydroxysafflor yellow A, lithospermic acid, and salvianolic acid $\mathrm{A}$ were obtained for further in vitro activity tests.

3.4. In Vitro Activity Tests for the Predicted Compounds. To confirm the ability of the hit compounds with TYR inhibitory activity, in vitro enzymatic activity assays were performed. Five concentrations of each compound were tested, and the results are shown in Table 4. As a well-known TYR inhibitor [5], kojic acid showed strong inhibition effect with a IC50 value of $127 \mu \mathrm{M}$. From the results shown in Figure 4, among the five identified hit compounds, protocatechuic aldehyde, hydroxysafflor yellow A, and tanshinone IIA possessed strong TYR inhibition effects in a dose-dependent manner, with the IC50 values of 455,498 , and $1214 \mu \mathrm{M}$, respectively. However, lithospermic acid and salvianolic acid A did not show 
<smiles>O=Cc1ccc(O)c(O)c1</smiles><smiles>O=C(O)C(O)Cc1ccc(O)c(O)c1</smiles><smiles>NC(Cc1ccccc1)C(=O)O</smiles>

13 Phenylalanine<smiles>Cc1coc2c1C(=O)C(=O)c1c-2ccc2c1CCCC2(C)C</smiles>

20 Tanshinone IIA

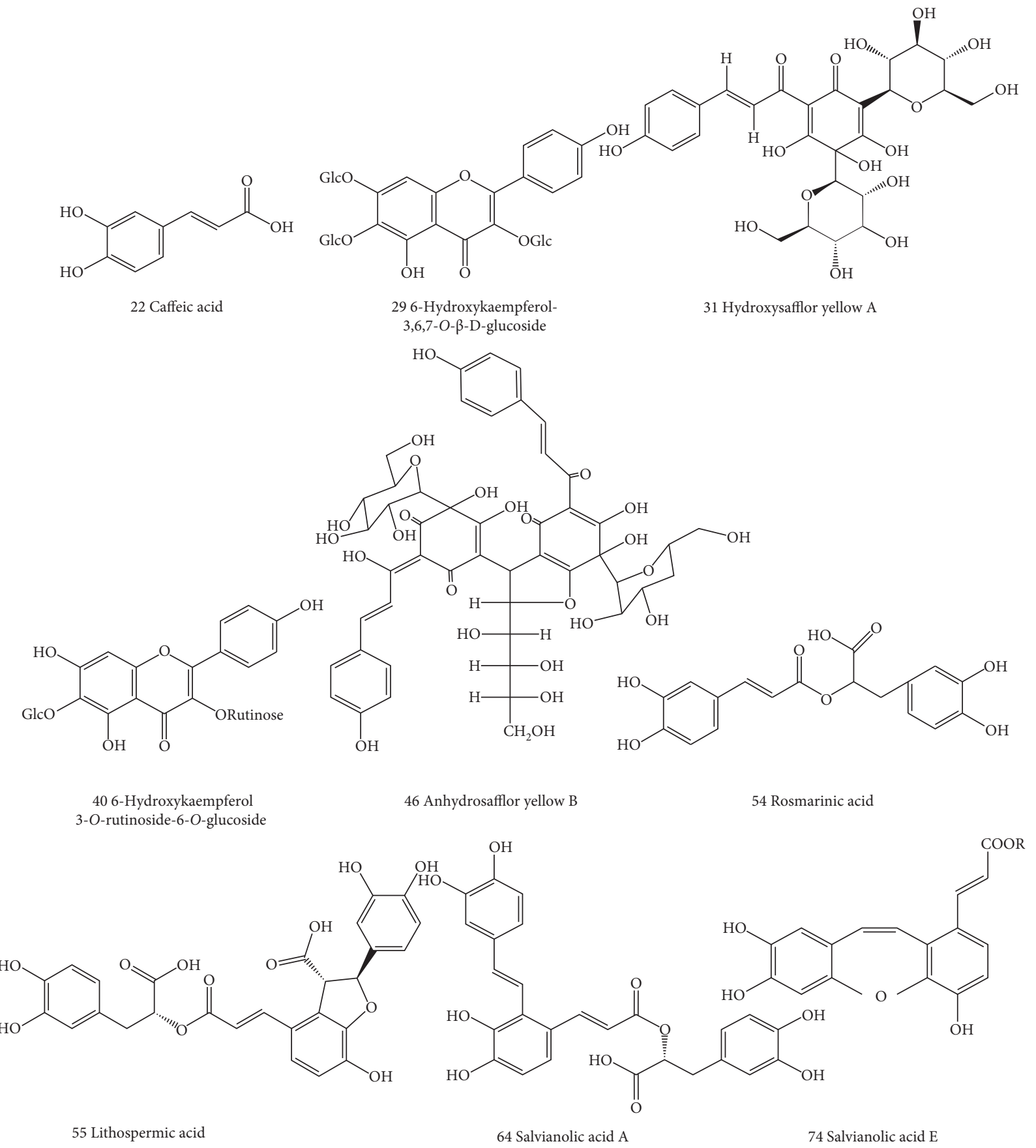

Figure 3: Chemical structures of compounds identified in DH herbal pair. The numbers of compounds are the same as the peak numbers in Figure 2. 
TABLE 4: Inhibition effect of protocatechuic aldehyde, tanshinone IIA, hydroxysafflor yellow A, lithospermic acid, and salvianolic acid A on tyrosinase $(n=9)$.

\begin{tabular}{|c|c|c|}
\hline Compounds & $\begin{array}{c}\text { Concentration } \\
(\mathrm{mmol})\end{array}$ & $\begin{array}{c}\text { Inhibition rate } \\
(\%)\end{array}$ \\
\hline Blank & PBS (50 mM, pH 6.8) & $0.44 \pm 0.02$ \\
\hline \multirow{5}{*}{ Kojic acid } & 1.6 & $88.93 \pm 0.28$ \\
\hline & 0.8 & $85.97 \pm 0.45$ \\
\hline & 0.4 & $76.63 \pm 1.02$ \\
\hline & 0.2 & $63.97 \pm 2.11$ \\
\hline & 0.1 & $40.72 \pm 0.15$ \\
\hline \multirow{5}{*}{$\begin{array}{l}\text { Protocatechuic } \\
\text { aldehyde }\end{array}$} & 1.6 & $80.18 \pm 0.08$ \\
\hline & 0.8 & $69.91 \pm 2.97$ \\
\hline & 0.4 & $49.80 \pm 1.30$ \\
\hline & 0.2 & $21.29 \pm 1.01$ \\
\hline & 0.1 & $13.78 \pm 0.95$ \\
\hline \multirow{5}{*}{ Tanshinone IIA } & 1.6 & $57.88 \pm 1.98$ \\
\hline & 0.8 & $37.26 \pm 2.87$ \\
\hline & 0.4 & $18.23 \pm 2.23$ \\
\hline & 0.2 & $6.03 \pm 1.12$ \\
\hline & 0.1 & $1.98 \pm 0.05$ \\
\hline \multirow{5}{*}{ Hydroxysafflor yellow A } & 1.6 & $62.48 \pm 1.34$ \\
\hline & 0.8 & $60.22 \pm 2.28$ \\
\hline & 0.4 & $55.85 \pm 1.80$ \\
\hline & 0.2 & $39.23 \pm 1.12$ \\
\hline & 0.1 & $14.58 \pm 0.55$ \\
\hline \multirow{5}{*}{ Lithospermic acid } & 1.6 & $26.09 \pm 3.28$ \\
\hline & 0.8 & $19.62 \pm 2.22$ \\
\hline & 0.4 & $11.52 \pm 2.64$ \\
\hline & 0.2 & $7.28 \pm 1.01$ \\
\hline & 0.1 & $4.32 \pm 0.21$ \\
\hline \multirow{5}{*}{ Salvianolic acid A } & 1.6 & $17.74 \pm 3.06$ \\
\hline & 0.8 & $14.42 \pm 1.14$ \\
\hline & 0.4 & $8.25 \pm 1.98$ \\
\hline & 0.2 & $5.04 \pm 0.91$ \\
\hline & 0.1 & $1.25 \pm 1.32$ \\
\hline
\end{tabular}

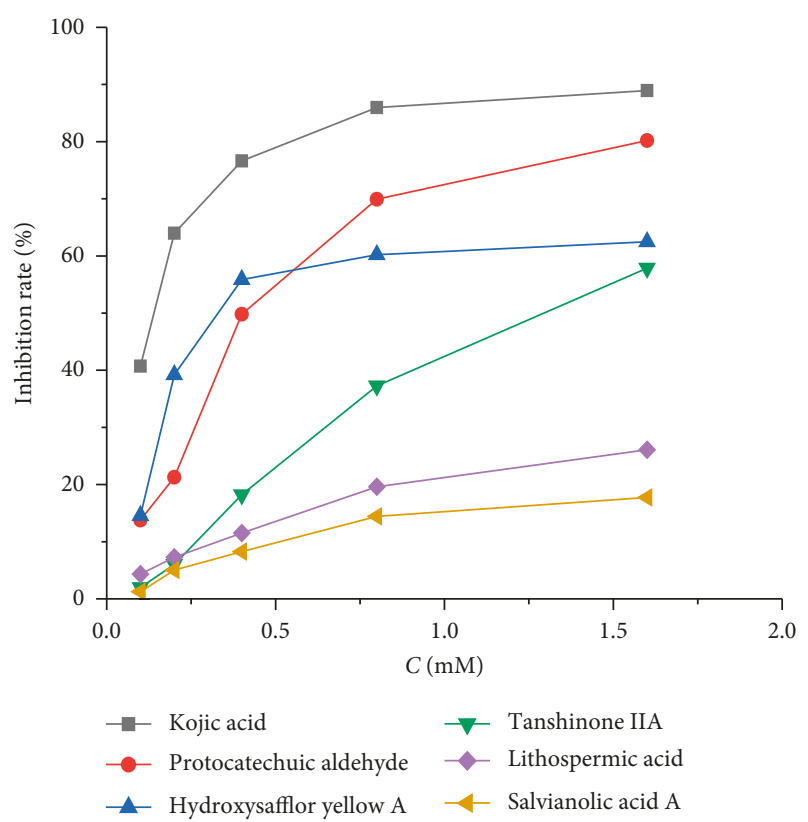

FIGURE 4: Inhibitory rate of active components to tyrosinase with different concentrations. significant inhibitory effect on tyrosinase at a relatively high concentration $(1.6 \mathrm{mM})$. The reason may be that they do not combine with the active amino acid residues or do not act on the active center of TYR, but further studies are required.

3.5. Molecular Docking of TYR and Identified Active Compounds. Molecular docking can be used to study the binding mechanism of compounds interacting with proteins. In this study, Autodock 4.2 was selected as the docking software to check out the active site of those components screened from DH extracts combined with TYR. The crystal structure of tyrosinase includes four identical parts, and one of them was used as the crystal structure of tyrosinase for computational docking analysis. Interestingly, as shown in Figures 5(a) and 5(b), protocatechuic aldehyde and hydroxysafflor yellow A could bond to the same catalytically active amino acid residues (THR324, ASN81, CYS83, GLU322, and HIS85) of TYR, which could explain the similar TYR inhibitory activity of these two compounds. Moreover, three hydrogen bonds (green line) between protocatechuic aldehyde, hydroxysafflor yellow $\mathrm{A}$, and the amino acid residues were observed. As shown in Figure 5(c), tanshinone IIA was found bonding into the hydrophobic cavity of tyrosinase (blue region) and surrounded by amino acid residues VAL248, HIS244, OTR410, VAL283, SER282, PRO277, PHE264, and ARG268 of tyrosinase. The amino acid residues of the tyrosinase to which the active compound binds are shown in Table 5. The reason for the weak activity may be that lithospermic acid and salvianolic acid A do not combine with the active amino acid residues (such as GLU322 and THR324) or do not act on the active center $\left(\mathrm{Cu}^{2+}\right)$ of tyrosinase. As tyrosinase is a copper-containing enzyme, it is expected that potential tyrosinase inhibitors should show high binding affinity for copper ions [26].

Along with the screening result, molecular docking was also carried out on compounds (absence of pure reference substances) having similar structure to protocatechuic aldehyde, lithospermic acid, and salvianolic acid A (the structure of these compounds are shown in Figure 4), attempting to explore other active TYR inhibitors by perspective of structure-activity relationship. As shown in Figure 5(a), the important region of protocatechuic aldehyde for copper chelation was the catechol structure. Compounds that functionally chelate copper ions at tyrosinase active sites have been frequently reported as effective tyrosinase inhibitors because they are analogous to the phenolic hydroxyl substrates of tyrosinase [27, 28]. In addition, danshensu, caffeic acid, rosmarinic acid, and salvianolic acid E having a catechol structure might also be potential TYR inhibitors. The docking result is shown in Table 5. With a portion of the same active sites with screened active compounds bound to TYR, it could be found that danshensu, caffeic acid, rosmarinic acid, salvianolic acid $\mathrm{E}$, anhydrosafflor yellow B, 6-hydroxykaempferol-3,6,7-O- $\beta$-D-glucoside, and 6hydroxykaempferol 3-O-rutinoside-6-O-glucoside similar to protocatechuic aldehyde, hydroxysafflor yellow A, and tanshinone IIA might also be potential TYR inhibitors. 


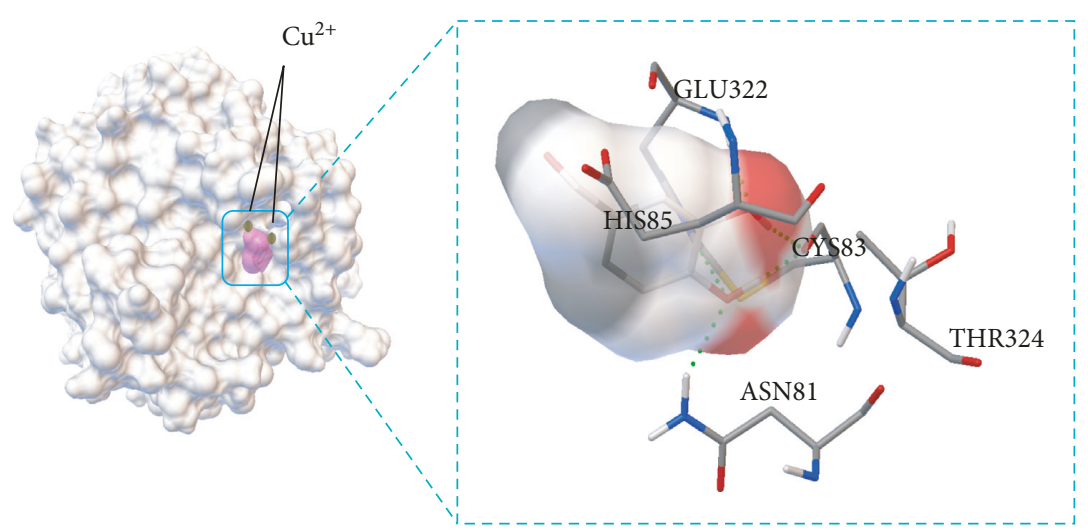

(a)

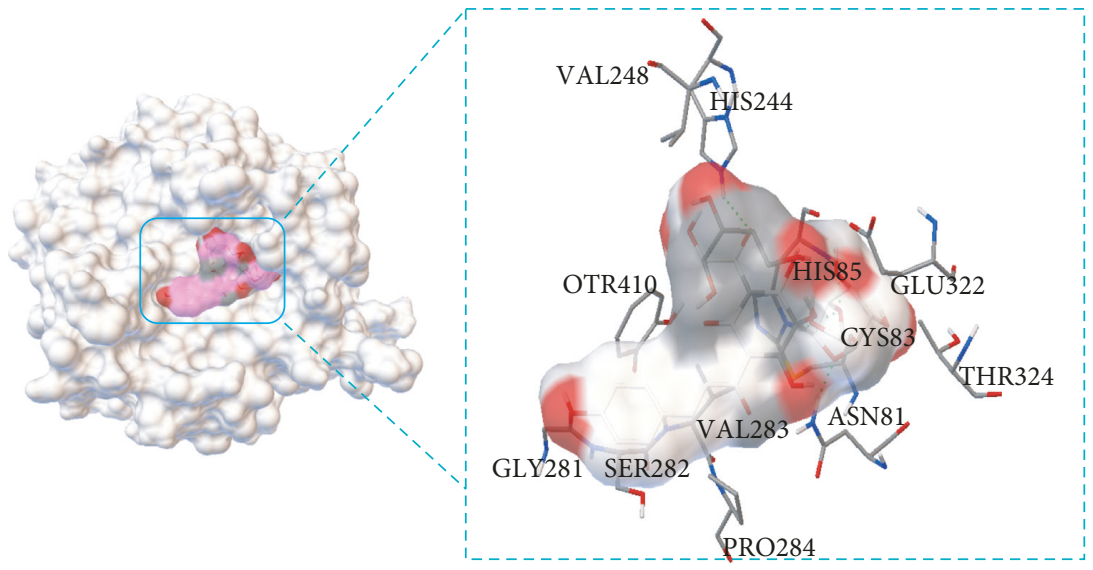

(b)

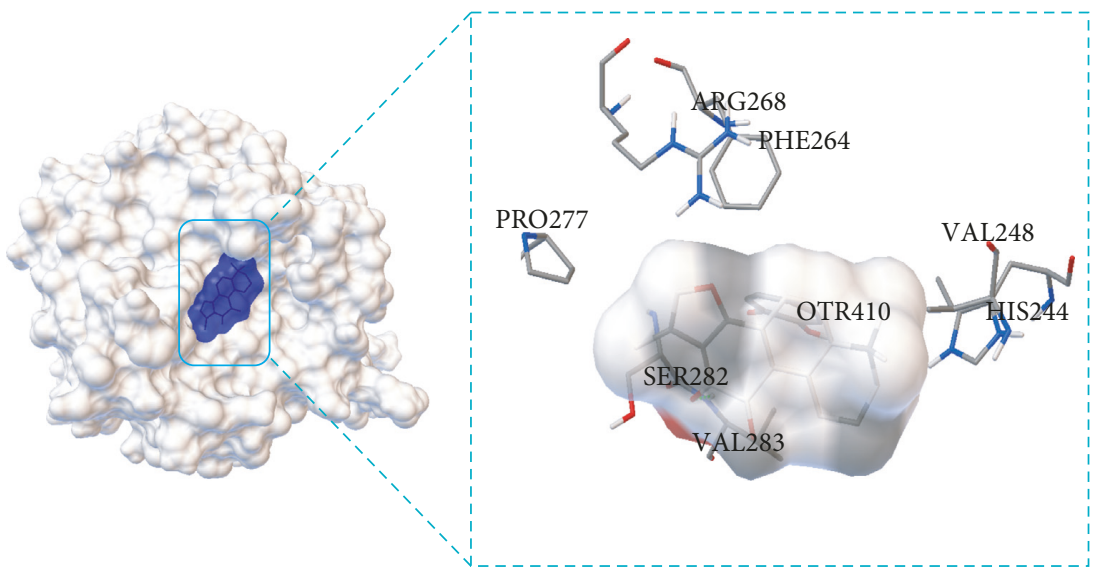

(c)

FIgURE 5: Molecular docking results of protocatechuic aldehyde (a), hydroxysafflor yellow A (b), and tanshinone IIA (c) with tyrosinase.

However, pure substances of these compounds were required for the further activity tests.

\section{Conclusions}

In this study, the TYR inhibitor screening methods were established, and the potential TYR inhibitory components from DH extract were screened. Combining the results of spectrum-effect analysis, LC-MS analysis, and enzymatic activity assay, three active compounds including protocatechuic aldehyde, hydroxysafflor yellow $\mathrm{A}$, and tanshinone
IIA were discovered as inhibitors targeting TYR. Meanwhile, docking results showed that these compounds might bind to the same amino acid residues in TYR catalytic pocket. Additionally, other potential active TYR inhibitors such as danshensu, caffeic acid, rosmarinic acid, and salvianolic acid E, which gained similar structures with the hit compounds, might also be identified. These results proved that the proposed method could effectively screen TYR inhibitors in complex mixtures and provided a reference for the discovery of other active TYR inhibitors. 
TABLE 5: Binding residues of identified compounds in DH herbal pair with tyrosinase.

\begin{tabular}{|c|c|c|}
\hline Compound & Residues & Residues with hydrogen bonding \\
\hline Kojic acid & $\begin{array}{l}\text { ASN81, GLU322, CYS83, THR324, THR84, } \\
\text { and HIS85 }\end{array}$ & ASN81, CYS83, and HIS85 \\
\hline Protocatechuic aldehyde & $\begin{array}{c}\text { THR324, ASN81, CYS83, GLU322, and } \\
\text { HIS85 }\end{array}$ & ASN81, CYS83, and HIS85 \\
\hline Hydroxysafflor yellow A & $\begin{array}{c}\text { THR324, ASN81, CYS83, GLU322, HIS85, } \\
\text { PRO284, VAL283, SER282, GLY281, } \\
\text { OTR410, and HIS244 }\end{array}$ & ASN81, CYS83, HIS85, and HIS244 \\
\hline Tanshinone IIA & $\begin{array}{l}\text { VAL248, HIS244, OTR410, VAL283, SER282, } \\
\text { PRO277, PHE264, and ARG268 }\end{array}$ & VAL283 and SER282 \\
\hline Lithospermic acid & $\begin{array}{c}\text { PRO270, THR261, GLY281, ARG268, } \\
\text { PHE264, ASN260, MET257, SER282, } \\
\text { VAL283, OTR410, VAL248, and HIS244 }\end{array}$ & $\begin{array}{l}\text { GLY281, ARG268, PHE264, SER282, and } \\
\text { VAL283 }\end{array}$ \\
\hline Salvianolic acid A & $\begin{array}{c}\text { ASN81, CYS83, HIS85, VAL283, SER282, } \\
\text { OTR410, HIS244, VAL248, PHE264, and } \\
\text { ARG268 }\end{array}$ & $\begin{array}{c}\text { CYS83, HIS85, VAL283, SER282, and } \\
\text { HIS244 }\end{array}$ \\
\hline Caffeic acid & $\begin{array}{l}\text { PHE264, ARG268, OTR410, GLY281, } \\
\text { VAL283, and SER282 }\end{array}$ & PHE264, ARG268, VAL283, and SER282 \\
\hline Danshensu & $\begin{array}{c}\text { PHE264, ARG268, OTR410, GLY281, } \\
\text { VAL283, and SER282 }\end{array}$ & ARG268, VAL283, and SER282 \\
\hline Phenylalanine & $\begin{array}{l}\text { HIS244, HIS85, THR84, CYS83, GLU322, } \\
\text { VAL283, and ASN81 }\end{array}$ & HIS85, THR84, and GLU322 \\
\hline Rosmarinic acid & $\begin{array}{c}\text { ARG268, PHE264, VAL248, HIS244, } \\
\text { GLU322, OTR410, HIS85, SER282, and } \\
\text { VAL283 }\end{array}$ & ARG268, GLU322, SER282, and VAL283 \\
\hline Salvianolic acid E & $\begin{array}{l}\text { ARG268, PHE264, VAL248, HIS244, HIS85, } \\
\text { SER282, VAL283, OTR410, and PRO284 }\end{array}$ & ARG268, SER282, VAL283, and HIS244 \\
\hline Anhydrosafflor yellow B & $\begin{array}{c}\text { GLU322, HIS244, HIS85, VAL248, VAL283, } \\
\text { PRO284, ASN81, OTR410, ASN260, SER282, } \\
\text { GLY281, PHE264, THR261, ARG268, and } \\
\text { PRO277 }\end{array}$ & GLY281 and ARG268 \\
\hline $\begin{array}{l}\text { 6-Hydroxykaempferol-3,6,7-O- } \beta \text {-D- } \\
\text { glucoside }\end{array}$ & $\begin{array}{c}\text { THR324, GLU322, ASN81, HIS85, HIS244, } \\
\text { OTR410, VAL283, PRO284, GLY281, and } \\
\text { SER282 }\end{array}$ & GLU322, ASN81, HIS85, and HIS244 \\
\hline $\begin{array}{l}\text { 6-Hydroxykaempferol 3-O-rutinoside-6-O- } \\
\text { glucoside }\end{array}$ & $\begin{array}{l}\text { THR324, ASN81, HIS85, OTR410, VAL283, } \\
\text { PRO284, GLY281, and SER282 }\end{array}$ & ASN81, HIS85, and GLY281 \\
\hline
\end{tabular}

\section{Data Availability}

The data used to support the findings of this study are available from the corresponding author upon request.

\section{Conflicts of Interest}

The authors declared that they have no conflicts of interest.

\section{Acknowledgments}

This work was supported by the National Natural Science Foundation of China (81703687 and 21275169) and the Science and Technology Research Program of Chongqing Municipal Education Commission (Grant no. KJ1709219). The authors sincerely acknowledge the Science and Technology Development Fund of Macao SAR and the University of Macau for financial support through the projects FDCT013-2015-A1 and MYRG2016-00144-ICMS-QRCM for this research.

\section{References}

[1] K. U. Zaidi, A. S. Ali, S. A. Ali, and I. Naaz, "Microbial tyrosinases: promising enzymes for pharmaceutical, food bioprocessing, and environmental industry," Biochemistry Research International, vol. 2014, Article ID 854687, 16 pages, 2014.

[2] W. M. Chai, M. K. Wei, R. Wang, R. G. Deng, Z. R. Zou, and Y. Y. Peng, "Avocado proanthocyanidins as a source of tyrosinase inhibitors: structure characterization, inhibitory activity, and mechanism," Journal of Agricultural and Food Chemistry, vol. 63, no. 33, pp. 7381-7387, 2015.

[3] M. Amer and M. Metwalli, "Topical hydroquinone in the treatment of some hyperpigmentary disorders," International Journal of Dermatology, vol. 37, no. 6, pp. 449-450, 1998.

[4] N. T. Madan Mohan, A. Gowda, A. K. Jaiswal et al., "Assessment of efficacy, safety, and tolerability of 4 -n-butylresorcinol $0.3 \%$ cream: an Indian multicentric study on melasma," Clinical, Cosmetic and Investigational Dermatology, vol. 9, pp. 21-27, 2016. 
[5] B. K. Singh, S. H. Park, H. B. Lee et al., "Kojic acid peptide: a new compound with anti-tyrosinase potential," Annals of Dermatology, vol. 28, no. 5, pp. 555-561, 2016.

[6] J. S. Roh, J. Y. Han, J. H. Kim, and J. K. Hwang, "Inhibitory effects of active compounds isolated from safflower (Carthamus tinctorius L.) seeds for melanogenesis," Biological and Pharmaceutical Bulletin, vol. 27, no. 12, pp. 1976-1978, 2004.

[7] C. Y. Chen, L. C. Lin, W. F. Yang, J. Bordon, and H. M. Wang, "An updated organic classification of tyrosinase inhibitors on melanin biosynthesis," Current Organic Chemistry, vol. 19, no. 1, pp. 4-18, 2015.

[8] R. Sarkar, P. Arora, and K. V. Garg, "Cosmeceuticals for hyper-pigmentation: what is available?," Journal of Cutaneous and Aesthetic Surgery, vol. 6, no. 1, pp. 4-11, 2013.

[9] X. Hu, J. W. Wu, M. Wang et al., "2-Arylbenzofuran, flavonoid and tyrosinase inhibitory constituents of Morus yunnanensis," Journal of Natural Products, vol. 75, no. 1, pp. 82-87, 2012.

[10] J. J. Zhu, G. R. Yan, Z. J. Xu et al., "Inhibitory effects of ( $\left.2^{\prime} R\right)-$ $2^{\prime}, 3^{\prime}$-dihydro-2' -(1-hydroxy-1-methylethyl)-2, $6^{\prime}$-bibenzofuran- $6,4^{\prime}$-diol on mushroom tyrosinase and melanogenesis in B16-F10 melanoma cells," Phytotherapy Research, vol. 29, no. 7, pp. 1040-1045, 2015.

[11] C. Qu, Z. J. Pu, G. S. Zhou et al., "Comparative analysis of main bio-active components in the herb pair DanshenHonghua and its single herbs by ultra high performance liquid chromatography coupled to triple quadrupole tandem mass spectrometry," Journal of Separation Science, vol. 40, no. 17, pp. 3392-3401, 2017.

[12] L. N. Gao, Y. L. Cui, K. Yan, and C. Qiu, “Advances in studies on compatibility of Salviae Miltiorrhizae Radix et Rhizoma and Carthami flos," Chinese Traditional and Herbal Drugs, vol. 47, pp. 671-679, 2016.

[13] J. K. No, M. S. Kim, Y. J. Kim, S. J. Bae, J. S. Choi, and H. Y. Chung, "Inhibition of tyrosinase by protocatechuic aldehyde," American Journal of Chinese Medicine, vol. 32, no. 1, pp. 97-103, 2004.

[14] Y. S. Chen, S. M. Lee, C. C. Lin, C. Y. Liu, M. C. Wu, and W. L. Shi, "Kinetic study on the tyrosinase and melanin formation inhibitory activities of carthamus yellow isolated from Carthamus tinctorius L.," Journal of Bioscience and Bioengineering, vol. 115, no. 3, pp. 242-245, 2013.

[15] A. Gunia Krzyzak, J. Popiol, and H. Marona, "Melanogenesis inhibitors: strategies for searching for and evaluation of active compounds," Current Medicinal Chemistry, vol. 23, no. 31, pp. 3548-3574, 2016.

[16] C. S. Zhu, Z. J. Lin, M. L. Xiao, H. J. Niu, and B. Zhang, "The spectrum-effect relationship a rational approach to screening effective compounds, reflecting the internal quality of Chinese herbal medicine," Chinese Journal of Natural Medicines, vol. 14, no. 3, pp. 177-184, 2016.

[17] Y. Y. Hou, X. L. Cao, L. Y. Dong et al., "Bioactivity-based liquid chromatography-coupled electrospray ionization tandem ion trap/time of flight mass spectrometry for $\beta 2 \mathrm{AR}$ agonist identification in alkaloidal extract of Alstonia scholaris," Journal of Chromatography A, vol. 1227, pp. 203-209, 2012.

[18] Q. F. Zheng, Y. L. Zhao, J. B. Wang et al., "Spectrum-effect relationships between UPLC fingerprints and bioactivities of crude secondary roots of Aconitum carmichaelii Debeaux (Fuzi) and its three processed products on mitochondrial growth coupled with canonical correlation analysis," Journal of Ethnopharmacology, vol. 153, no. 3, pp. 615-623, 2014.
[19] L. M. Zhuo, J. J. Peng, Y. L. Zhao et al., "Screening bioactive quality control markers of QiShenYiQi dripping pills based on the relationship between the ultra-high performance liquid chromatography fingerprint and vascular protective activity," Journal of Separation Science, vol. 40, no. 20, pp. 4076-4084, 2017.

[20] M. A. Soares, M. A. Almeida, C. Marins Goulart, O. A. Chaves, A. Echevarria, and M. C. C. de Oliveira, "Thiosemicarbazones as inhibitors of tyrosinase enzyme," Bioorganic and Medicinal Chemistry Letters, vol. 27, no. 15, pp. 3546-3550, 2017.

[21] G. M. Morris, R. Huey, W. Lindstrom et al., "AutoDock4 and AutoDockTools4: automated docking with selective receptor flexibility," Journal of Computational Chemistry, vol. 30, no. 16, pp. 2785-2791, 2009.

[22] Y. J. Wang, G. W. Zhang, J. K. Yan, and D. M. Gong, "Inhibitory effect of morin on tyrosinase: insights from spectroscopic and molecular docking studies," Food Chemistry, vol. 163, pp. 226-233, 2014.

[23] D. W. Banner and P. Hadvary, "Crystallographic analysis at 3.0-a resolution of the binding to human thrombin of four active site-directed inhibitors," Journal of Biological Chemistry, vol. 266, pp. 20085-20093, 1991.

[24] J. Cao, J. J. Xu, X. G. Liu, S. L. Wang, and L. Q. Peng, "Screening of thrombin inhibitors from phenolic acids using enzyme-immobilized magnetic beads through direct covalent binding by ultrahigh-performance liquid chromatography coupled with quadrupole time-of-flight tandem mass spectrometry," Journal of Chromatography A, vol. 1468, pp. 86-94, 2016.

[25] X. X. Yang, F. Xu, D. Wang et al., "Development of a mitochondria-based centrifugal ultrafiltration/liquid chromatography/ mass spectrometry method for screening mitochondria-targeted bioactive constituents from complex matrixes: herbal medicines as a case study," Journal of Chromatography A, vol. 1413, pp. 33-46, 2015.

[26] L. S. Chaves, M. C. C. de Barros, C. M. R. de Oliveira et al., "Biological interactions of fluorinated chalcones: stimulation of tyrosinase activity and binding to bovine serum albumin," Journal of Fluorine Chemistry, vol. 199, pp. 30-38, 2017.

[27] I. E. Orhan and M. T. H. Khan, "Flavonoid derivatives as potent tyrosinase inhibitors-a survey of recent findings between 2008 and 2013," Current Topics in Medicinal Chemistry, vol. 14, no. 12, pp. 1486-1493, 2014.

[28] Y. J. Kim and H. Uyama, "Tyrosinase inhibitors from natural and synthetic sources: structure, inhibition mechanism and perspective for the future," Cellular and Molecular Life Sciences, vol. 62, no. 15, pp. 1707-1723, 2005. 

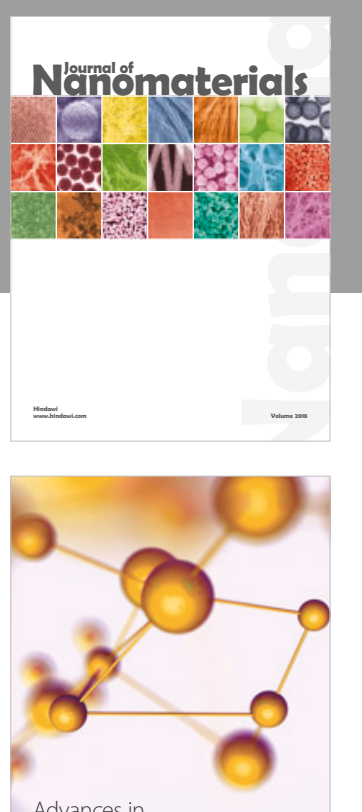

Physical Chemistry
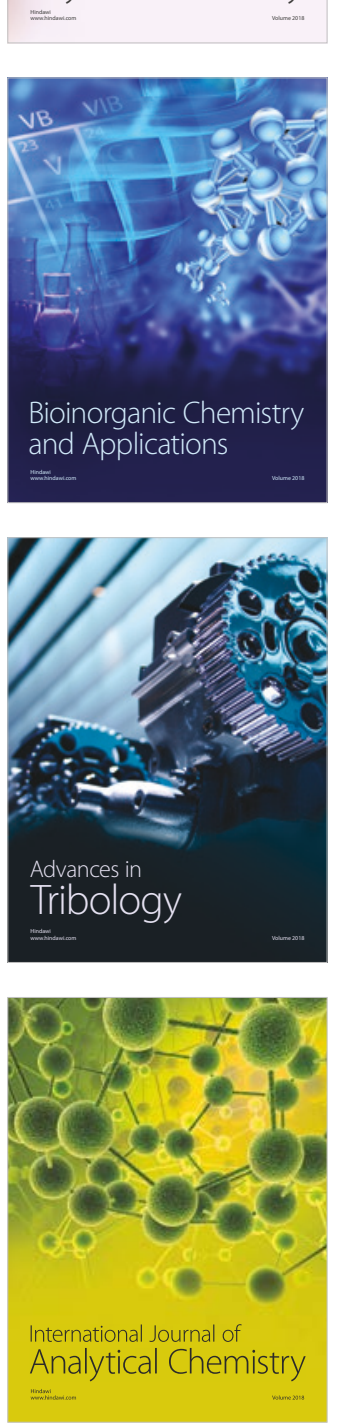

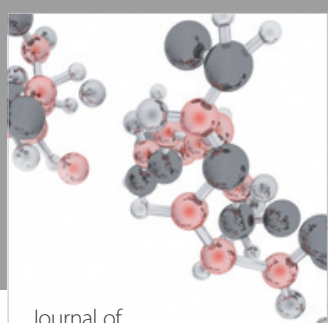

Analytical Methods

in Chemistry

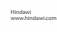

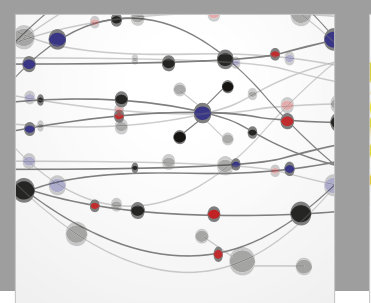

The Scientific World Journal

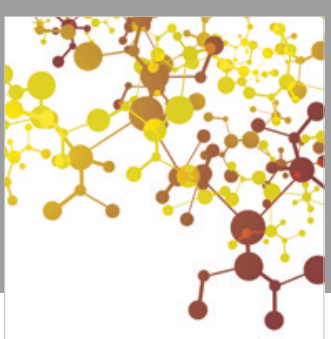

Journal of

Applied Chemistry
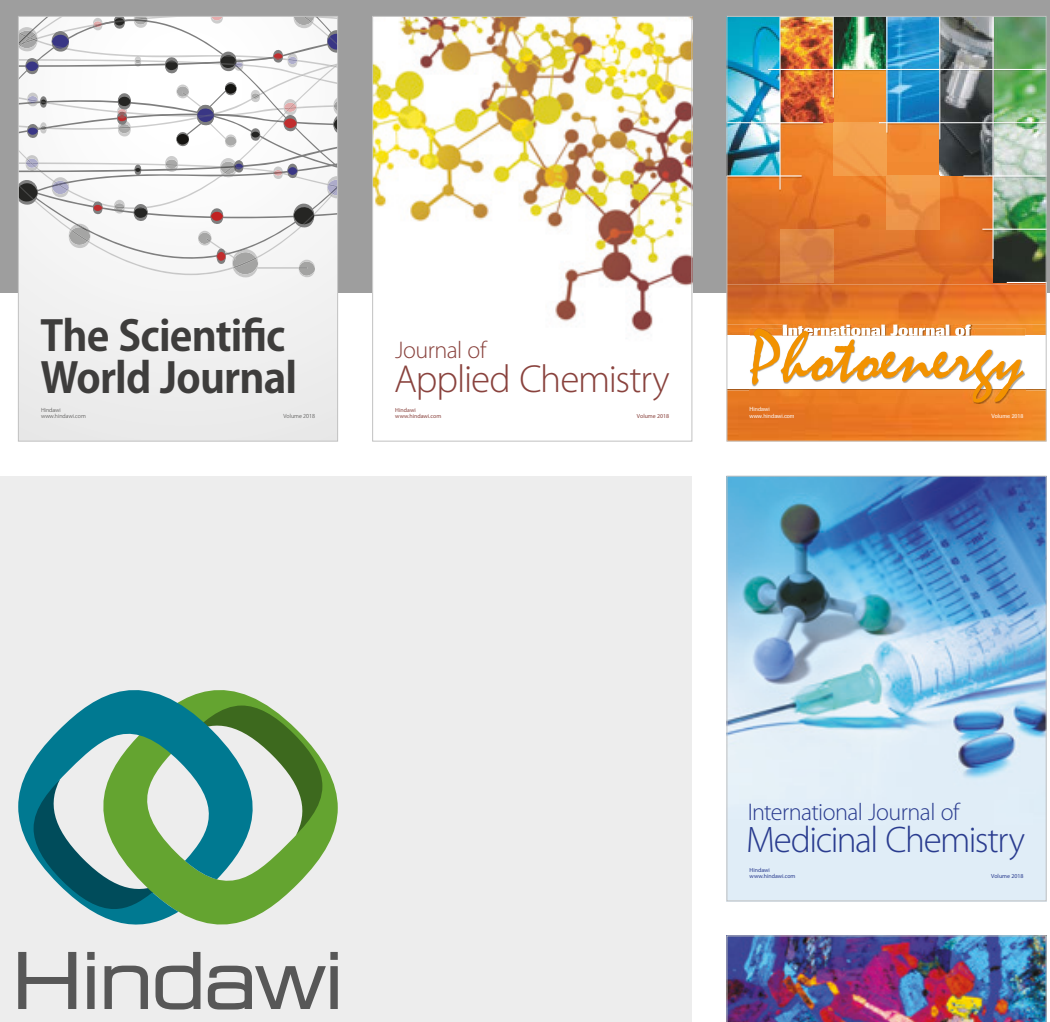

Submit your manuscripts at

www.hindawi.com
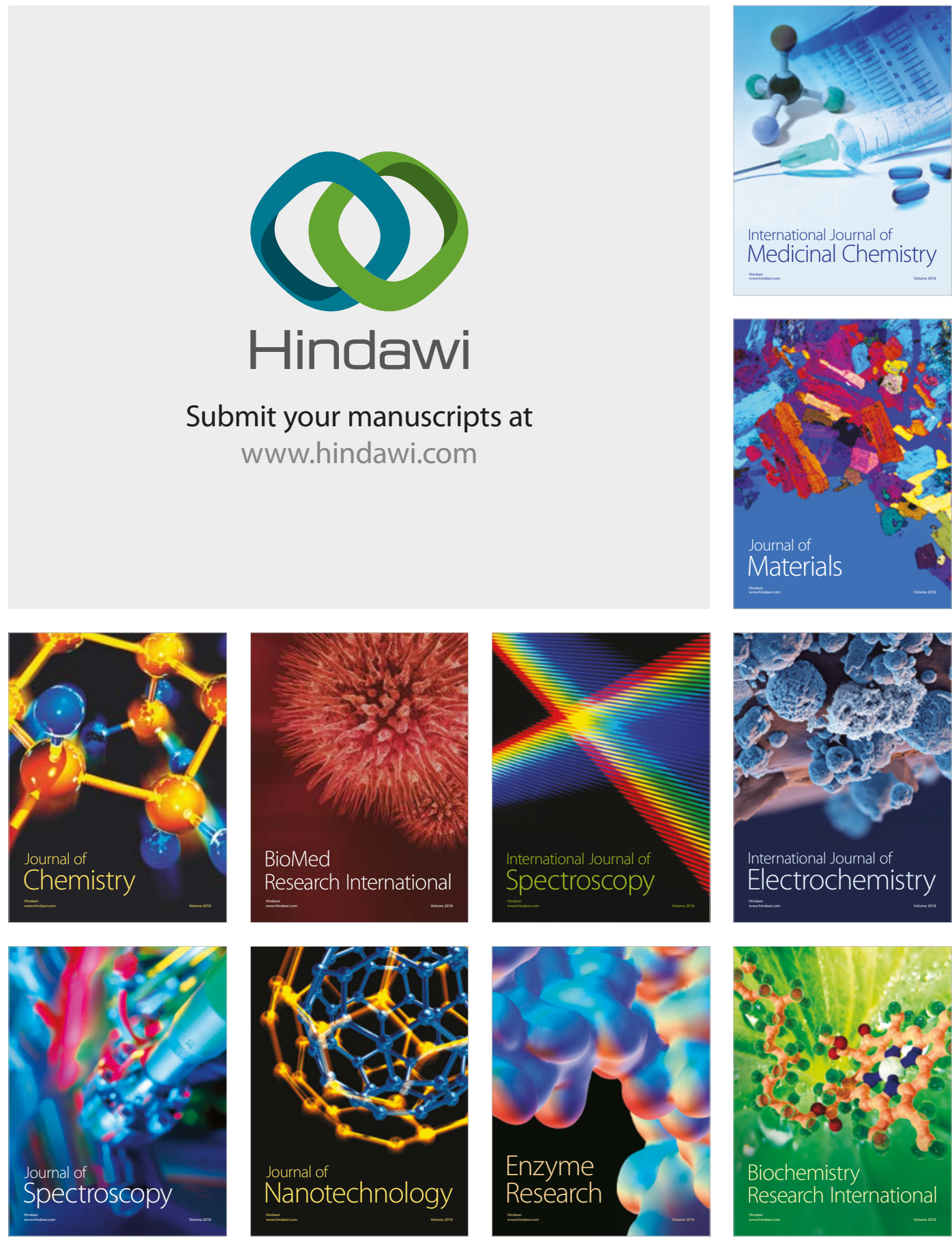
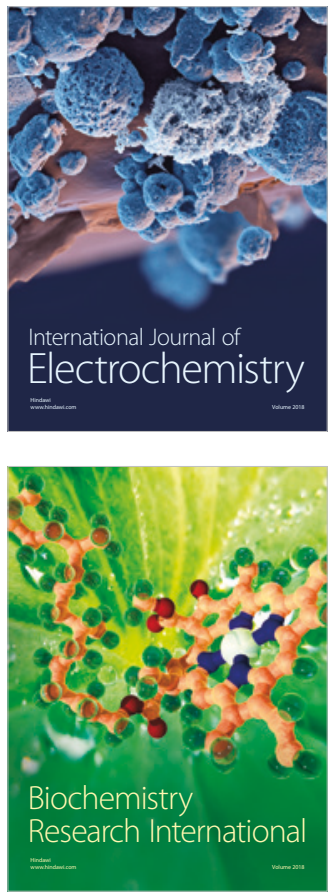